\title{
Infectious causes of fever of unknown origin
}

\author{
Authors: Alastair C McGregor ${ }^{A}$ and David A Moore ${ }^{B}$
}

The causes of fever of unknown origin (FUO) are changing because advances in clinical practice and diagnostics have facilitated the identification of some infections. A variety of bacterial infections can cause FUO, and these can be divided into those that are easy to identify using culture and those that require serological or molecular tests for identification. A number of viral, parasitic and fungal infections can also cause prolonged fever. This article summarises the clinical features and diagnostic strategy of these infections.

\section{Introduction}

Many infectious agents can cause a chronic, low-grade fever but, with improvements in diagnostic technology, some of these are now easy to identify, provided that the correct tests are performed. This means that they comprise a lower proportion of fever of unknown origin (FUO) than previously. Current estimates of the proportion of FUO that is caused by infection ranges between 15 and $30 \%$ in temperate regions. ${ }^{1,2}$ This figure greatly varies between populations, reflecting geographical region, population age and type of medical practice.

The infections discussed below all have the potential to cause prolonged illness but, given the ease of diagnosis of some, not all commonly cause classical FUO. Chronic infections can be categorised according to the type of organism. Bacterial causes can be subdivided into culturable organisms causing focal occult infection (osteomyelitis, occult abscess and bacterial endocarditis) and intracellular organisms that are technically impossible to culture but that can be diagnosed using serological tests (eg Rickettsia, Coxiella or Bartonella spp). In between these extremes are a group of infections caused by bacteria that may be difficult but not impossible to culture, which can cause chronic infection of a more insidious nature (enteric fever, tuberculosis, brucellosis and yersiniosis). Parasitic causes of chronic fever include leishmaniasis and malaria. Infectious mononucleosis (caused by Epstein-Barr virus (EBV), cytomegalovirus (CMV) or Toxoplasma) may uncommonly cause a fever lasting more than three weeks. In addition, endemic fungi, which can occasionally cause

Authors: ${ }^{A}$ SpR, Hospital for Tropical Diseases, London, UK, and Imported Fever Service, RIPL, Public Health England, Porton Down,

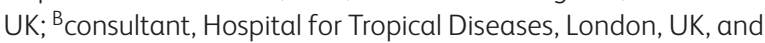
London School of Hygiene and Tropical Medicine, London UK

\section{Table 1. Diagnostic tests for infections.}

\begin{tabular}{ll}
$\begin{array}{l}\text { Type of infection } \\
\text { Bacterial }\end{array}$ & Diagnosis \\
Endocarditis & Imaging and bacterial culture \\
Occult abscess & Imaging and bacterial culture \\
Osteomyelitis & Imaging and bacterial culture \\
Enteric fever & Culture of blood and stool \\
Tuberculosis & Imaging and tissue culture \\
Brucellosis & Imaging and bacterial culture \\
Yersiniosis & Culture and serology \\
Coxiellosis & Serology and PCR \\
Bartonellosis & Serology \\
Rickettsiosis & Serology and PCR \\
Viruses & \\
CMV & Serology \\
EBV & Serology \\
Parasites & \\
Malaria & Blood film, $\mathrm{PCR}$ and dipstick \\
Leishmania & Culture, $\mathrm{PCR}$ and histology \\
Toxoplasma & Serology \\
Fungi & Culture at $30^{\circ} \mathrm{C}$ and $37^{\circ} \mathrm{C}$ serology \\
Histoplasma & $30^{\circ} \mathrm{C}$ and $37^{\circ} \mathrm{C}$ serology \\
Blastomyces & $30^{\circ} \mathrm{C}$ and $37^{\circ} \mathrm{C}$ serology \\
Coccidioides & Culture at \\
& \\
\hline
\end{tabular}

deep-seated mycoses, while extremely uncommon in the UK, should be considered in travellers. Diagnostic tests for these infections are summarised in Table 1.

It is important to recognise that the range of opportunistic pathogens that can present with FUO is considerably more extensive in immunosuppressed individuals, such as those with HIV, chronic renal impairment or transplant recipients, and patients receiving treatment with corticosteroids or biologics. A thorough medical history that captures potentially localising symptoms, possible exposure to pathogens (travel, occupation etc) and susceptibility to infection (prophylaxis, vaccination, previous medical and surgical history, and immune status) will help in identifying possible infections and forming an appropriate testing strategy. 


\section{Bacterial infections}

\section{Focal occult infections}

Infection with almost any bacterial pathogen can cause a febrile illness without localisation. However, relatively few of these infections can persist for three weeks without either spontaneous resolution or deterioration to the point that the infection can be localised or become detectable on routine investigations. Small abscesses with low-grade bacterial pathogens can cause focal infection that is difficult to localise but can be identified with imaging. Classical examples would be subphrenic abscesses, empyema, osteomyelitis or prostatic infection. There are few specific symptoms associated with bacterial endocarditis but only a low-grade pathogen (such as a HACEK organism or a viridans streptococcus) would be likely to escape detection for three weeks. The isolation of these organisms is not usually a challenge but inappropriate administration of antibiotics can suppress bacterial growth in blood cultures.

\section{Unculturable bacterial infection}

Bartonella, Coxiella and Rickettsia spp are all capable of causing indolent infection and none of these bacteria can be cultured using standard microbiological techniques. Since the identification of these organisms relies on the request and performance of specific serological or molecular tests, a high degree of suspicion is required to diagnose them. The different species of Bartonella have widely differing epidemiologies and the most commonly seen infection in the UK is catscratch fever. Coxiella causes Q fever which is associated with exposure to animal carcasses, although because the organism is so highly infectious (eg may be carried on dust cloud) this link can be very tenuous or inapparent. Rickettsia spp, which causes spotted fevers and typhus, are arthropod-borne and are generally imported into the UK.

\section{Key points}

Fever of unknown origin (FUO) can result from infection with a variety of bacteria, viruses, parasites and fungi.

The diagnosis of a bacterial infection can often be made on culture of suitable samples but may require the detection of specific antibodies or bacterial DNA for organisms that are difficult to grow.

The identification of viral, parasitic and fungal infections may require serological, molecular or histological tests.

Cross-sectional imaging is useful for identifying foci of infection which may then be sampled for further testing.

A thorough history with emphasis on medical history, travel, exposure, prophylaxis, vaccination and immune status is important when considering likely infectious causes of FUO.

KEYWORDS: Fever, pyrexia, chronic, unknown, origin

\section{Enteric fever}

Typhoidal Salmonella can cause a grumbling fever without localising features. Typically, the fever is initially low grade, rises in the second week, and resolves by the fourth week in untreated, nonfatal infection. ${ }^{3}$ Diagnosis is made on isolation of Salmonella typhi or Salmonella paratyphi from stool, urine or blood, although culture of blood is only about $65 \%$ sensitive and empiric therapy may well be required. ${ }^{4}$

\section{Tuberculosis}

Infection with Mycobacterium tuberculosis is a common cause of FUO, particularly with extrapulmonary localisation (eg skeletal, renal). The symptoms of active tuberculosis are primarily caused by the immune response to a relatively low burden of organism and this fact, coupled with the slow replication time of the organism, means that proving a diagnosis of tuberculosis with culture or molecular tests can be impossible. Cross-sectional imaging may aid the diagnosis of tuberculosis. Ultimately, diagnosis of tuberculosis in challenging cases may rely on a trial of therapy.

\section{Brucellosis}

Infection with Brucella spp often causes low-grade pyrexia. Brucellosis is a multi-organ disease and there may or may not be localising features. The disease can resemble tuberculosis. There are usually clues in the clinical history (geographical, exposure to goats and camels or unpasteurised milk). Diagnosis is made on culture or serology.

\section{Yersiniosis}

The enteropathogenic species of Yersinia, Yenterocolitica and Y paratuberculosis, can cause chronic febrile illnesses. As the nomenclature suggests, classical presentation of infection with these organisms is enterocolitis and adenitis (primarily mesenteric), respectively. The organism is widespread in nature but infections are very uncommon in the UK and often occur in outbreaks. Diagnosis can be made on culture (selective agar may increase sensitivity of stool culture) or serological testing. ${ }^{5}$

\section{Infectious mononucleosis}

Primary infection with EBV or CMV in adults can cause an infectious mononucleosis (IM) syndrome, characterised by lymphadenopathy, fever and the appearance of atypical mononuclear cells in the peripheral blood. About $80 \%$ of cases of IM are caused by EBV, with most of the remaining cases associated with acute CMV infection. ${ }^{6}$ Fever can persist for as long as 35 days in cases of acute CMV. Acute infection with Toxoplasma gondii can also cause an infectious mononucleosislike syndrome, although this probably counts for no more than $1 \%$ of the total number of cases of IM. ${ }^{7}$

\section{Parasitic infections}

Visceral leishmaniasis and malaria are the only common parasitic causes of FUO. Visceral leishmaniasis is caused by members of the Leishmania donovani complex. There are 
two species within this complex: L infantum (also known as $L$ chagasi), which has a distribution from the Mediterranean to China, as well as parts of South America, and L donovani in India and East Africa. The infection is spread by sandflies and the presentation is usually insidious, with low-grade fever, hepatosplenomegaly and anorexia with wasting. Diagnosis is made by serology or demonstration of parasites in tissue (usually bone marrow) using microscopy, culture or PCR.

Malaria usually causes a more acute febrile illness but infection with the 'benign' species of Plasmodium ie $P$ malariae, ovale and vivax, may occasionally present with recurrent fever over weeks. $P$ ovale and $P$ vivax form hepatic 'hypnozoite' states, and recrudescence may occur many years after infection.

\section{Fungal infections}

Endemic fungi are characterised by their temperature dimorphism (ie they exist as different fungal forms at different temperatures), their restricted geographical distribution and their ability to cause disease in healthy individuals. ${ }^{8}$ Pulmonary or disseminated infection with fungi of the genera Histoplasma, Blastomyces, Coccidiodes and Paracoccidiodes can cause chronic febrile symptoms and, although extremely uncommon in travellers returning to the UK, should be considered in those with an appropriate geographical exposure.

\section{Conclusion}

A wide variety of infections can cause chronic pyrexia but, with improved diagnostics, some classical causes of FUO are now easy to identify. In addition to bacterial culture, serological and molecular tests may be used to look for specific pathogens. Cross-sectional and echographic imaging techniques may be used to identify tissue abnormalities which can then be sampled to provide a pathological diagnosis in challenging cases of FUO.

\section{References}

1 Efstathiou SP, Pefanis AV, Tsiakou AG et al. Fever of unknown origin: discrimination between infectious and non-infectious causes. Eur J Intern Med 2010 Apr;21:137-43.

2 Bleeker-Rovers CP1, Vos FJ et al. A prospective multicenter study on fever of unknown origin: the yield of a structured diagnostic protocol. Medicine (Baltimore) 2007;86:26-38.

3 Pegues DA, Miller SI. Salmonella species, including Salmonella Typhi. In: Mandell GL, Bennett JE, Dolin R (eds), Mandell, Douglas, and Bennett's principles and practice of infectious disease, 7th ed, vol 2. Philadelphia, PA: Churchill Livingstone, 2009:2887-903.

4 Farooqui BJ1, Khurshid M, Ashfaq MK, Khan MA. Comparative yield of Salmonella typhi from blood and bone marrow cultures in patients with fever of unknown origin. J Clin Pathol 1991;44:258-9.

5 Dennis DT, Mead PS (2010). Yersinia species, including plague. In: Mandell GL, Bennett JE, Dolin R (eds), Mandell, Douglas, and Bennett's principles and practice of infectious disease, 7th ed, vol 2. Philadelphia, PA: Churchill Livingstone, 2009:2943-53.

6 Klemola E, Von Essen R, Henle G, Henle W. Infectiousmononucleosis-like disease with negative heterophil agglutination test. Clinical features in relation to Epstein-Barr virus and cytomegalovirus antibodies. J Infect Dis 1970;121:608-14.

7 Remington JS, Barnett CG, Meikel M, Lunde MN. Toxoplasmosis and infectious mononucleosis. Arch Intern Med 1962;110:744-53.

8 Kauffman CA. Endemic mycoses: blastomycosis, histoplasmosis, and sporotrichosis. Infect Dis Clin North Am 2006;20:645-62,vii.

Address for correspondence: Dr A McGregor, Hospital for Tropical Diseases, 235 Euston Road, London NW1 2BU, UK. Email: alastair.mcgregor@uclh.nhs.uk

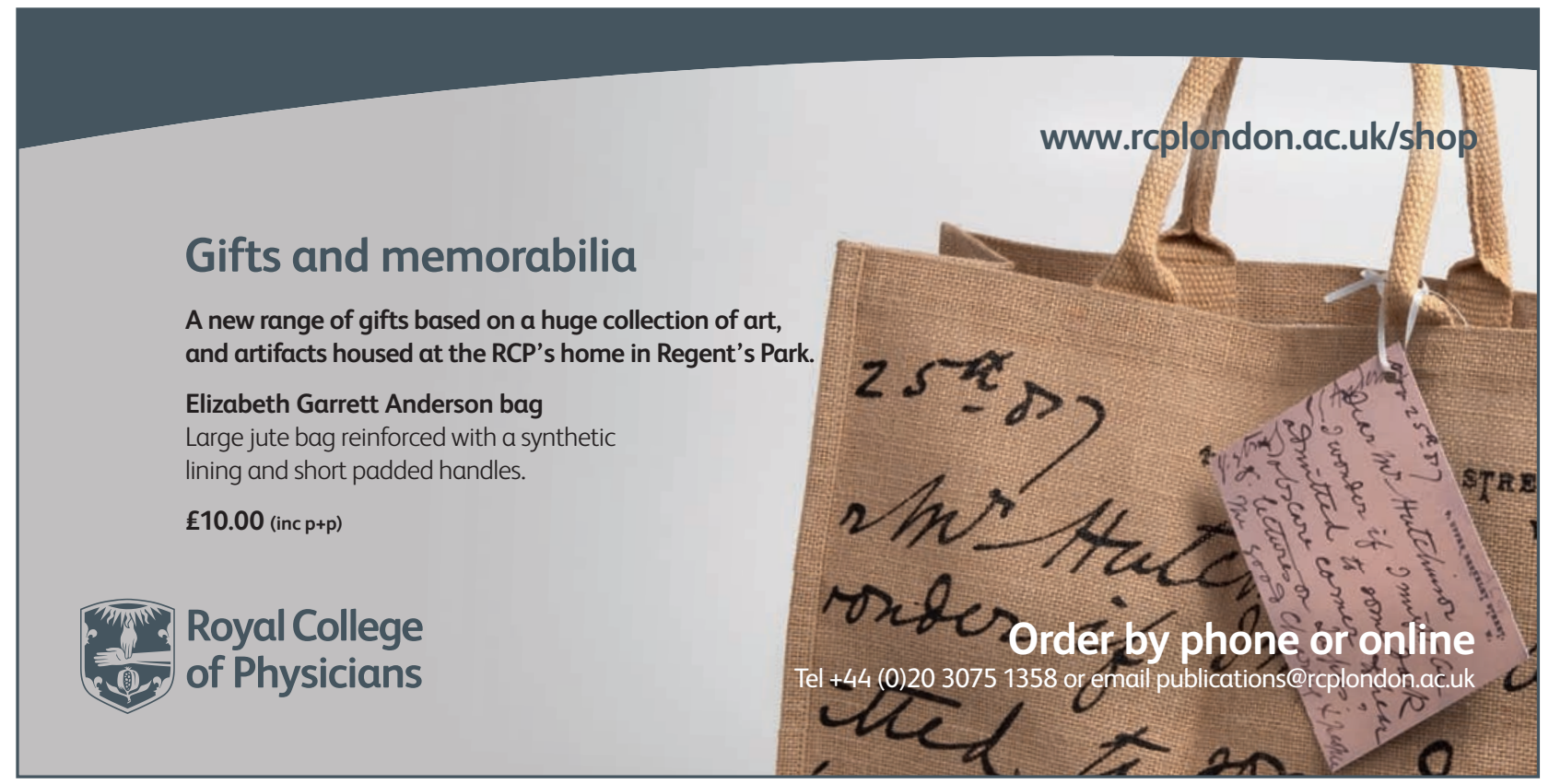

